\title{
Association of VEGF Gene Family Variants with Central Macular Thickness and Visual Acuity after Aflibercept Short-Term Treatment in Diabetic Patients: A Pilot Study
}

\author{
Ahmed A. Abdelghany ${ }^{a}$ Eman A. Toraih ${ }^{b, c}$ Ahmed A. Mohamed ${ }^{d}$ \\ Rehab M. Lashine $^{\mathrm{e}}$ Mai H.S. Mohammad ${ }^{f}$ Mohamed S. Nafie ${ }^{g}$ \\ Manal S. Fawzy h, i \\ aDepartment of Ophthalmology, Faculty of Medicine, Suez Canal University, Ismailia, Egypt; ${ }^{\text {bDepartment of }}$ \\ Surgery, Tulane University, School of Medicine, New Orleans, LA, USA; 'Department of Histology and Cell Biology \\ (Genetics Unit), Faculty of Medicine, Suez Canal University, Ismailia, Egypt; ${ }^{d}$ Department of Ophthalmology,

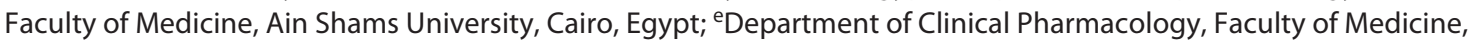 \\ Suez Canal University, Ismailia, Egypt; ' Department of Clinical Pathology, Faculty of Medicine, Suez Canal University, \\ Ismailia, Egypt; ${ }^{9}$ Department of Chemistry, Faculty of Science, Suez Canal University, Ismailia, Egypt; ${ }^{\text {hDepartment }}$ \\ of Medical Biochemistry and Molecular Biology, Faculty of Medicine, Suez Canal University, Ismailia, Egypt; \\ 'Department of Biochemistry, Faculty of Medicine, Northern Border University, Arar, Saudi Arabia
}

\section{Keywords}

Aflibercept · Diabetic retinopathy · Genetic polymorphisms . Real-time polymerase chain reaction - Vascular endothelial growth factor

\begin{abstract}
Introduction: Diabetic retinopathy (DR) is one of the major vision-threatening causes worldwide. Searching for an individualized therapeutic strategy to prevent its progress is challenging. Objective: This work aimed to investigate the association of angiogenesis-inducer vascular endothelial growth factor (VEGF) gene family and related receptor variants (rs833069, rs12366035, rs7664413, rs7993418, and rs2305948) with susceptibility of DR and the response to 1 dose of aflibercept treatment in type 2 diabetes mellitus (T2DM). Methods: Consecutive eligible patients with T2DM $(n=125)$ and 110 unrelated controls were enrolled in this
\end{abstract}

preliminary prospective case-controlled study. Genotyping was identified using TaqMan real-time PCR. Adjusted odds ratio (OR) with $95 \%$ confidence interval $(\mathrm{Cl})$ was applied to assess the strength of the association with the clinical/ophthalmological characteristics and early response to intravitreal aflibercept treatment in terms of improved visual acuity (BCVA) and central macular thickness (CMT). Results: We found that both VEGFB rs12366035 and VEGFC rs7664413 conferred higher risk for DR progression under allelic (OR [95\% Cl]: 1.71 [1.07-2.74]), homozygote comparison (3.55 [1.32-9.57]), and recessive (3.77 [1.43-9.93]) models for the former and under allelic (2.09 [1.25-3.490, homozygote comparison (2.76 [1.02-7.45]), and recessive (2.62 [0.986.98] models for the latter. In contrast, VEGFR1 rs7993418 conferred protection against DR under heterozygote comparison and dominant models. The rs12366035*T/T genotype showed the worst pretreatment BCVA score $(0.35 \pm$ $0.24)$ compared to other corresponding genotypes ( $0.66 \pm$ karger@karger.com

www.karger.com/ore

Karger ${ }^{\prime}=$
(C) 2020 S. Karger AG, Basel
Manal S. Fawzy

Department of Biochemistry and Molecular Biology

Faculty of Medicine, Suez Canal University

Ring road, Ismailia 41522 (Egypt)

manal2_khashana@ymail.com 
0.26 in $C / T$ and $0.54 \pm 0.25$ in C/C carriers) ( $p=0.008)$. Meanwhile, patients with rs $7993418^{*} \mathrm{G} / \mathrm{G}$ of VEGFR1 exhibited a significant reduction in CMT after aflibercept injection (12.26 $\pm 35.43 \mu$ in G/G vs. $3.57 \pm 8.74 \mu$ in $\mathrm{A} / \mathrm{A})(p=0.037)$. Conclusions: Polymorphisms of the studied VEGF/receptors could be considered as genetic risk factors of DM/DR development and could play an important role in aflibercept early response for DR patients in the study population.

(c) 2020 S. Karger AG, Basel

\section{Introduction}

As a key player in the growing burden of diabetes mellitus (DM), diabetic macular edema (DME) is the leading cause of sight-threatening and avoidable blindness worldwide in general and in the developing countries in particular [1]. This "angiogenesis-driven eye disease" is characterized by a retinal thickening in the macular area due to the breakdown of the blood-retinal barrier and accumulation of extracellular fluid in the intraretinal area or in the subretinal space. In addition, in more severe cases, it occurs as cystoid edema with a typical petaloid appearance [1] (Fig. 1a). A primary concern of DME is inflammation, with the involvement of multiple cytokines and chemokines, including the potent vasopermeability and angiogenesis-promoting vascular endothelial growth factor (VEGF) [1] (Fig. 1b). Previous research studies recognized the critical role played by this factor in DME pathogenesis and the direct correlation its levels showed with the severity of macular edema [2]. Therefore, over the past decade, the literature has emerged that offer supporting findings of the most effective strategies for the treatment of DME using frequent intravitreal (IV) injections of anti-VEGF drugs [2-5].

Ranibizumab (Lucentis; Genentech, San Francisco, CA, USA), bevacizumab (Avastin; Genentech), and aflibercept (Eylea/VEGF Trap; Regeneron Pharmaceuticals, Tarrytown, NY, USA) were the most popular antiVEGF drugs shown to produce efficacy in phase III clinical trials [6-8]. In related reports, the Diabetic Retinopathy Clinical Research Network (DRCR.net) found that aflibercept provided superior visual recovery if baseline visual acuity (VA) was poorer than 69 ETDRS letters (approximately $6 / 15$ Snellen) when compared to the other anti-VEGF agents [6].

Aflibercept (DrugBank No. 08885), which received FDA approval in 2014 for the treatment of DME, is a humanized recombinant fusion protein. It consists of fragments from the extracellular domains of human VEGF receptors 1 and 2 fused to the Fc portion of human IgG1 (https:// pubchem.ncbi.nlm.nih.gov/substance/124490314) (Fig. 1c). Having a higher binding affinity to VEGFA and VEGFB compared to other antiVEGF medications, it is supposed to have a longer biological activity (lasts up to 10-12 weeks) with subsequently improved pharmacokinetics and decreased the frequency of usage (every 2 months) which can be cost saving [9-11].

Although the selection of the drug of choice, the calculation of the accurate dose, and the implementation of the treatment protocol vary greatly according to the primary response of the DME patients to anti-VEGF drugs, many patients showed an either inconsistent or transient response to treatment apart from the degree of retinal ischemia, the course of the disease, and the associated systemic conditions [12]. This issue has sparked interest in genetic association studies to identify the putative loci, which could be implied in treatment response [1].

Several VEGF single-nucleotide polymorphisms (SNPs) were investigated for their link to the DME susceptibility and/or response to treatment in several populations [13-17]. However, to the best of our knowledge, no single study was conducted to validate the association of the 5 selected variants of VEGF and its receptors collectively with the susceptibility and/or treatment outcomes of aflibercept in a sample of Middle East population. To address this issue, we carried out this work to assess the putative utility of the specified SNPs as diagnostic and predictive genetic biomarkers in diabetic retinopathy (DR) patients.

Fig. 1. Role of the VEGF pathway and ocular anti-VEGF therapy in DR. a Pathological features in DR. b Putative action of the VEGF pathway of DR. c Structure and function of aflibercept drug. Aflibercept is a recombinant fusion protein composed of 2 main portions: VEGF-binding domains from the extracellular domains of human VEGFR1 and VEGFR2 receptors, which are then fused to the Fc portion of human IgG1. It acts as a decoy receptor for the ligands VEGFA, VEGFB, and PIGF with a greater affinity than the body's native receptors. It prevents these ligands from binding to endothelial receptors (VEGFR-1 and VEGFR-2), which in turn will suppress neovascularization, decrease vascular permeability, and ultimately slow vision loss. $\mathbf{d}$ OCT of a good responder DR before and after intravitreal injection of aflibercept. Before treatment (left photos), OCT showed cystoid macular edema and serous macular detachment with a CMT of $489 \mu \mathrm{m}$. After treatment (right photos), OCT showed marked resolution of macular edema with CMT of $293 \mu \mathrm{m}$ with $196-\mu \mathrm{m}$ reduction in macular edema posttreatment. VEGF, vascular endothelial growth factor; DR, diabetic retinopathy; PIGF, placental growth factor; OCT, optical coherence tomography; CMT, central macular thickness.

(For figure see next page.) 


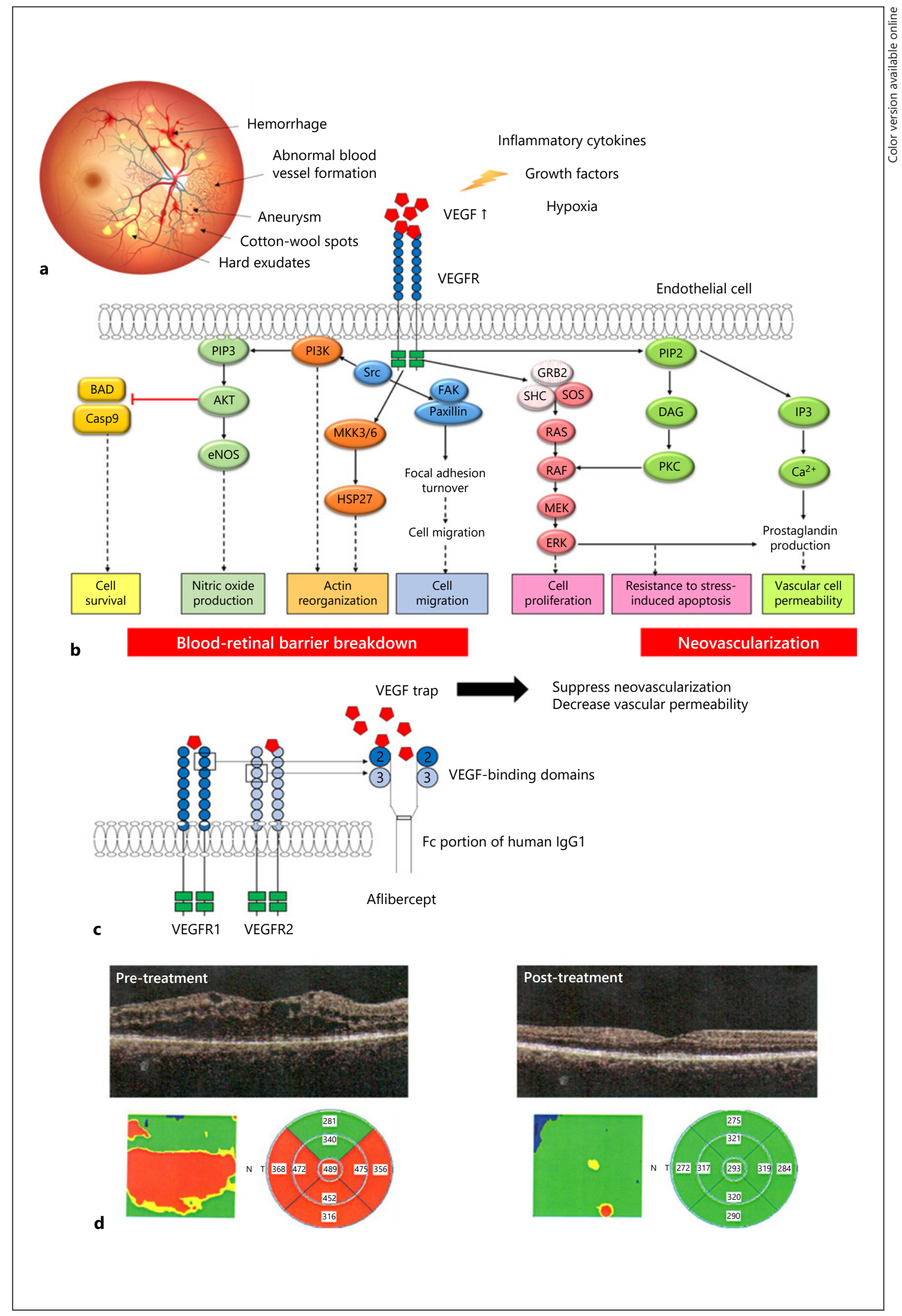

VEGF Gene Family Variants and Diabetic

Ophthalmic Res 2021;64:261-272 


\section{Patients and Methods}

\section{Study Participants}

The present case-control study was conducted on 125 consecutive patients complaining of type 2 diabetes mellitus (T2DM) who attended the Ophthalmology Outpatient Department of Suez Canal University Hospitals and a private clinic, Ismailia, Egypt, during the period between November 2018 and the end of February 2019. Patients with T2DM were assigned according to the Early Treatment Diabetic Retinopathy Study (ETDRS) design report [18] into DR subgroup $(n=80)$ and non-DR subgroup $(n=45)$. About 42.5 and $22 \%$ of DR and non-DR patients were on insulin therapy, respectively (online suppl. Table 1; for all online suppl. material, see www.karger.com/doi/10.1159/000511087). Patients with "other ocular diseases (including neovascular glaucoma, agerelated macular degeneration (AMD), or rhegmatogenous retinal detachment), vasculopathy other than DR (including retinal vein occlusion or retinal vasculitis), history of ocular trauma or surgery, and chronic disease other than T2DM (e.g., liver or kidney disorders and autoimmune disease)" were excluded. The control group $(n=110)$ included participants presented in the same period of patient recruitment for driving license check or pre-employment check with normal glucose tolerance. The study was approved by the institutional research ethics committee of the Faculty of Medicine, Suez Canal University (Approval No. 3648), and conducted according to the principles of the Declaration of Helsinki. We obtained written informed consent from all the study participants before the start of the study.

\section{Clinical Evaluation}

All patients were subjected to a thorough clinical ophthalmic evaluation and optical coherence tomography (OCT). Ophthalmic examination at the first presentation and the follow-up period included best-corrected visual acuity (BCVA) - using Snellen's chart and converted to log of the Minimum Angle of Resolution (logMAR) unit for further statistical analysis - anterior segment examination using slit-lamp biomicroscopy, posterior segment examination using both $+20 \mathrm{D}$ and $+90 \mathrm{D}$ lenses, colored fundus photography, and OCT. Based on the fundus fluorescein angiography, which has been performed at the first presentation for the DR group, the type of maculopathy and classification (focal vs. diffuse type) have been assessed by the ophthalmologist [18]. The ischemic type was excluded to avoid unreliable results.

DR grading into nonproliferative DR (NPDR) and proliferative DR (PDR) was performed according to the ETDRS. Subsequently, NPDR was subdivided into mild, moderate, and severe. The diagnosis of macular edema is based on both clinical and OCT findings. Clinically significant DME was defined as "any retinal thickening within $500 \mu \mathrm{m}$ of the center of the fovea, hard exudates within 500 $\mu \mathrm{m}$ of the center of the fovea with adjacent retinal thickening, and retinal thickening at least one disc diameter in size which is within one disc diameter of the center of the fovea" [18].

Optical Coherence Tomography Scans

OCT scan for all patients' retinas was performed to determine the "central macular thickness (CMT)." OCT of the macula was performed using OPTOVUE RTVue XR (OPTOVUE Inc., Fremont, CA, USA). All scans were performed through clear media after the dilatation of the pupil. RTVue scans used the macular scan protocol set to $6 \times 6 \mathrm{~mm}$ containing the main 12 clock hour scans, horizontal, longitudinal, and oblique scans, evaluated with OPTOVUE analysis software version \# 6, 3, 2, and 73. Also, a fast macular thickness map centered on the fovea was performed.

OCT findings of DME included central subfield thickness (CST) $>290 \mu \mathrm{m}$ together with spongy intraretinal edema. Cases with interrupted inner segment/outer segment (IS/OS) junction or interrupted external limiting membrane were excluded as they carry a poor prognosis that will yield invalid results.

All patients with DME underwent IV injection using $2 \mathrm{mg}$ in $0.05 \mathrm{~mL}$ of aflibercept (Eylea $40 \mathrm{mg} / \mathrm{mL}$; Bayer Pharma AG, Berlin, Germany), and blood samples were taken just before injection. The detailed IV injection procedure and follow-up of the patients were elaborated in our previous work on the same patient cohort [19].

Both BCVA and OCT were performed again after 4 weeks of the IV injection to evaluate the CMT (Fig. 1d). In line with the current aim to assess the association of the study genetic variants with susceptibility of DR and the early response to 1 dose of aflibercept, improvement of BCVA in terms of $>2$ lines of the Snellen's chart (converted to $\log$ MAR units for the statistical analysis) and reduction in the CST by $>15 \%$ of the pretreatment thickness were considered as a response to treatment. Patients' follow-up continued in regular visits according to the DR follow-up schedule, and reinjection was scheduled when indicated. Both BCVA and CMT changes in all treated patients during the follow-up period were calculated and recorded.

\section{Allelic Discrimination PCR of VEGF Variants}

Blood samples $(5 \mathrm{~mL})$ were drawn from the median cubital vein of participants under aseptic conditions on EDTA tubes after overnight fasting. Genomic DNA was extracted from the buffy coat using the QIAamp DNA Blood Mini kit (Catalog No. 51104; Qiagen) following the manufacturer's instructions. DNA concentration and purity were assessed using NanoDrop ND-1000 (NanoDrop Technologies Inc., Wilmington, DE, USA). The VEGF and related receptor variants (rs833069, rs12366035, rs7664413, rs7993418, and rs2305948) were genotyped using StepOne Real-Time PCR (Applied Biosystems, Foster City, CA, USA). Genotyping was performed blinded to the case/control status. PCR reactions were run in duplicates in a total volume of 25 $\mu \mathrm{L}$ containing genomic DNA (20 ng) diluted to $11.25 \mu \mathrm{L}$ with nuclease-free water, $1.25-\mu \mathrm{L}$ predesigned TaqMan-based SNP Genotyping Assay (20×) working stock (assay ID:C_11400863_20 for rs833069, C_31727614_10 for rs12366035, C_11780939_10 for rs7664413, C_1910654_10 for rs7993418, or C_22271999_20 for rs2315948), and $12.5-\mu \mathrm{L}$ TaqMan genotyping PCR Master Mix, no UNG (4440043) with $100 \%$ concordance rate for genotype calls. No-template negative controls were run with each experiment to assure there was no evidence of contamination. The following thermocycler conditions were followed: denaturation at $95^{\circ} \mathrm{C}$ for $10 \mathrm{~min}$, followed by 40 cycles of $90^{\circ} \mathrm{C}$ for $15 \mathrm{~s}$ and $60^{\circ} \mathrm{C}$ for $1 \mathrm{~min}[20]$.

\section{Ligand-Receptor Interaction Assessment}

This type of in silico analysis aimed to explore whether the study variants will affect the corresponding receptor binding sites' activity and their biological function using an approach that highlights the binding site amino acids and the virtual binding mechanism of the cocrystallized ligands with the critical amino acids inside the receptor binding sites. Our molecular modeling (visualization) studies for VEGF family genes were carried out using 
Table 1. Ophthalmological assessment of diabetic retinopathy patients according to their grade

\begin{tabular}{|c|c|c|c|c|c|c|c|c|}
\hline$n$ & 80 & 24 & 31 & 11 & 14 & & & \\
\hline Age, years & $59.8 \pm 9.3$ & $57.4 \pm 5.8$ & $57.6 \pm 9.9$ & $63.5 \pm 8.5$ & $65.9 \pm 10.4^{\dagger, \neq}$ & 0.010 & 0.384 & $<0.001$ \\
\hline Sex - female & $54(67.5)$ & $17(70.8)$ & $23(74.2)$ & $7(63.6)$ & $7(50.0)$ & 0.426 & 0.137 & 0.225 \\
\hline Hypertension & $56(70.0)$ & $13(54.2)$ & $20(64.5)$ & $10(90.9)$ & $13(92.9)$ & 0.028 & 0.322 & 0.004 \\
\hline Onset, years & $43.9 \pm 9.9$ & $47.2 \pm 7.2$ & $43.1 \pm 11.3$ & $45.7 \pm 7.3$ & $38.4 \pm 10.4 \dagger$ & 0.053 & -0.283 & 0.011 \\
\hline Insulin users & $34(42.5)$ & $3(12.5)$ & $12(38.7)$ & $8(72.7)$ & $11(78.6)$ & $<0.001$ & 0.502 & $<0.001$ \\
\hline Pre-CMT, $\mu \mathrm{m}$ & $413 \pm 139$ & $443 \pm 174$ & $407 \pm 130$ & $380 \pm 108$ & $400 \pm 110$ & 0.597 & -0.061 & 0.593 \\
\hline Post-CMT, $\mu \mathrm{m}$ & $333 \pm 105$ & $335 \pm 96$ & $337 \pm 116$ & $293 \pm 55$ & $349 \pm 129$ & 0.597 & -0.038 & 0.737 \\
\hline$\Delta \mathrm{CMT}, \mu \mathrm{m}$ & $-80 \pm 109$ & $-107 \pm 147$ & $-69 \pm 92$ & $-87 \pm 83$ & $-51 \pm 109$ & 0.427 & 0.029 & 0.800 \\
\hline Pre-BCVA & $0.51 \pm 0.25$ & $0.36 \pm 0.26$ & $0.46 \pm 0.16$ & $0.61 \pm 0.23^{\dagger}$ & $0.80 \pm 0.15^{\dagger, \ddagger}$ & $<0.001$ & 0.565 & $<0.001$ \\
\hline Post-BCVA & $0.34 \pm 0.24$ & $0.22 \pm 0.19$ & $0.30 \pm 0.15$ & $0.33 \pm 0.20$ & $0.67 \pm 0.23^{\dagger, 末,} \S$ & $<0.001$ & 0.550 & $<0.001$ \\
\hline
\end{tabular}

Data are shown as $n(\%)$ or mean $\pm \mathrm{SD}$. The $\chi^{2}$ test was used for qualitative variables, and the Mann-Whitney U test and Student's $t$ test were employed for quantitative variables and represented by $p$ ass values. Spearman's rank test was used for correlation analysis between the stages of diabetic retinopathy and the clinical variables, represented as a correlation coefficient ( $\mathrm{r})$ and $p$ value $(p$ corr). $p$ value $<0.05$ was considered as statistically significant. PDR, proliferative diabetic retinopathy; NPDR, nonproliferative diabetic retinopathy (mild, moderate, and severe); responders, improved 1 month classified by $\triangle \mathrm{CMT}$; $\triangle \mathrm{CMT}$, change in CMT; CMT, central macular thickness; Pre, pretreatment with aflibercept; Post, posttreatment with aflibercept; BCVA, best-corrected visual acuity assessed by the logarithm of the minimum angle of resolution. $p$ values $<0.05$ are considered significant. ${ }^{\dagger}$ Compared to mild NPDR. ${ }^{\ddagger}$ Compared to moderate NPDR. ${ }^{\S}$ Compared to severe NPDR.

Chimera software [21]. The chosen receptors were $4 \mathrm{KZN}, 2 \mathrm{C} 7 \mathrm{~W}$, 2X1W, 5EW3, and 1RV6 for VEGFA, VEGFB, VEGFC, VEGFR2, and VEGFR1 genes, respectively. The Protein Data Bank (PDB)coded receptors were freely accessed (https://www.rcsb.org/). Both the receptors and the ligands were optimized using Maestro software. Binding site disposition and analysis of ligand-receptor interactions were carried out by analysis of hydrogen bond and lipophilic interactions according to the Medicinal Chemist's Guide to Molecular Interactions [22].

\section{Statistical Analysis}

Statistical analysis was carried out using R version 3.5.3 and Statistical Package for the Social Sciences (SPSS) for windows version 22. Sample size calculations were performed using G*Power software version 3.1.9.4. The Kolmogorov-Smirnov test was applied for data distribution and normality assessment. The HardyWeinberg equilibrium (HWE) and allele and genotype frequencies of the studied variants were estimated within each group, as mentioned previously [23]. Adjusted odds ratio (OR) and 95\% confidence interval (CI) for multiple genetic association models were calculated using SNPStats (https://www.snpstats.net/start.htm). For quantitative variables, the one-way ANOVA test for parametric attributes and Mann-Whitney or Kruskal-Wallis tests for nonparametric data were executed. For qualitative variables, the $\chi^{2}$ test was used. The correlation was calculated using the Spearman correlation test, and the matrix was plotted using the "corrplot" package. $p$ value $<0.05$ was considered significant.

\section{Results}

\section{Baseline Characteristics of the Study Population}

A total of 235 individuals were enrolled in the current study: 125 diabetic patients (91 women and 34 men) with a mean age of $61.1 \pm 8.8$ and 110 control subjects $(75$ women and 35 men) with a mean age of $59.1 \pm 5.9(p=$ 0.475 for sex and 0.051 for age). On the comparison between patients with retinopathy and without retinopathy, the former group showed higher mean age $(p=0.035)$, later age at onset $(p<0.001)$, and shorter disease duration $(p=0.001)$ (online suppl. Table 1$)$.

\section{Ophthalmological Assessment of Diabetic Retinopathy Patients}

Patients with higher grades of retinopathy showed associations with advanced age $(p=0.010)$, shorter disease duration $(p<0.001)$, hypertension $(p=0.028)$, and insulin usage $(p<0.001)$. Moreover, baseline and posttreatment VA were the worst among the PDR group $(p<$ 0.001) (Table 1).

DR patients were then classified according to the improvement of delta CMT to anti-VEGF therapy into responders (63 patients) and nonresponders (17 patients). 
Table 2. Comparison of genotype and allele frequencies in patients and controls

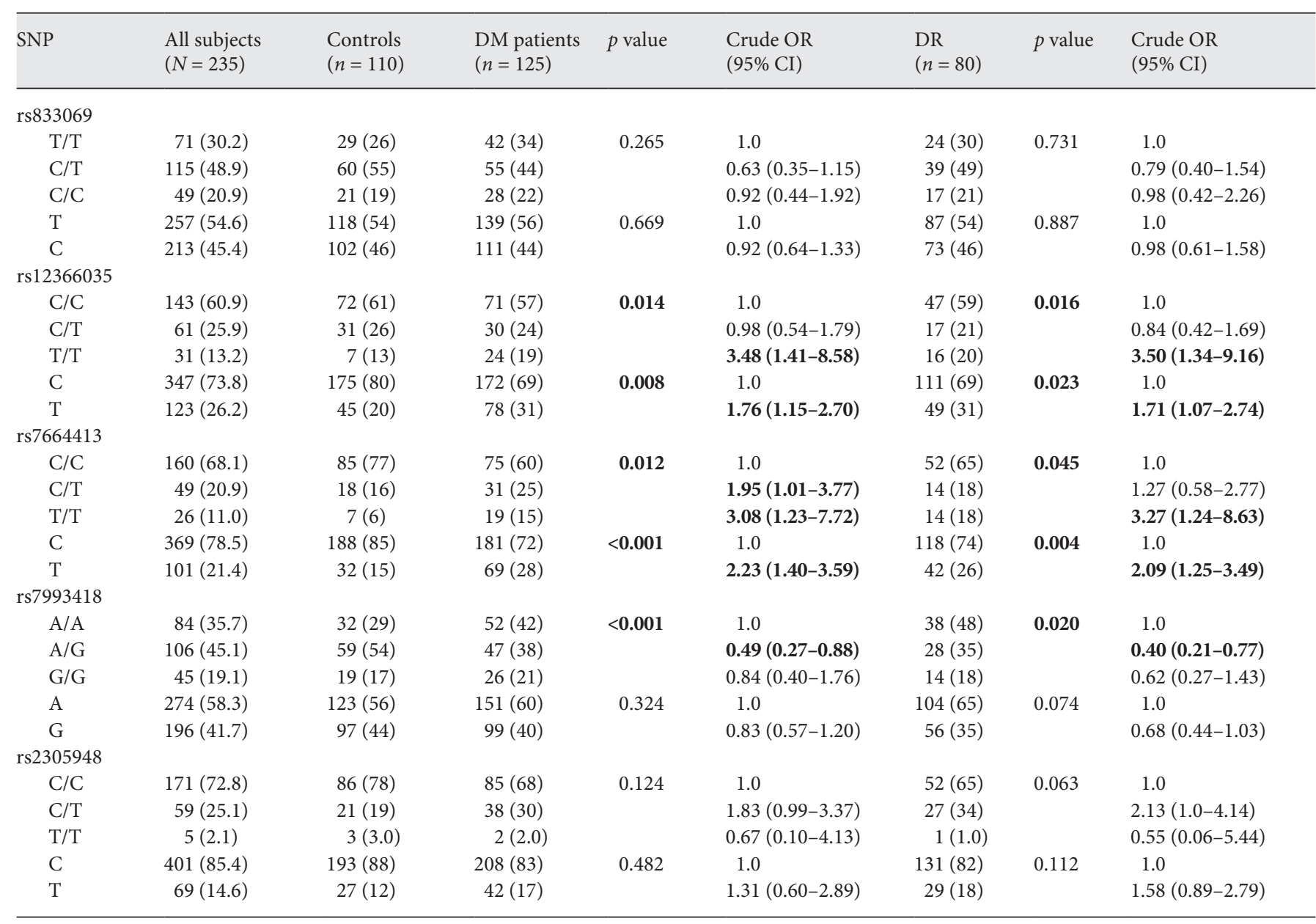

Data are shown as $n(\%)$. The two-sided $\chi^{2}$ test was used. Patients with DM included 45 cohorts without DR and 80 subjects with DR. Bold values indicate significance at $p$ value $<0.05$. DM, diabetes mellitus; DR, diabetic retinopathy; OR, odds ratio; 95\% CI, 95\% confidence interval.

The responder group exhibited higher baseline CMT (433 $\pm 145 \mu \mathrm{m})$ compared to the nonresponder group (337 \pm $76 \mu \mathrm{m})(p=0.008)$. No other significant differences were found between the 2 patient cohorts regarding demographic and clinical parameters (online suppl. Table 2).

Correlation analysis is depicted in online suppl. Figure 1. Disease duration and DR grade were directly correlated with pretreatment BCVA $(r=0.61$ and $r=0.64, p<0.001)$ and posttreatment BCVA ( $r=0.56$ and $r=0.67, p<$ $0.001)$, respectively. In addition, higher age was positively correlated with hypertension $(r=0.59, p<0.001)$.

\section{Allelic Discrimination of VEGF Gene Family Variants in the Study Groups}

Genotype frequencies of rs833069 (T/C) of VEGFA and rs2305948 (C/T) of VEGFR2 polymorphisms were following those expected by the HWE in both patients and controls $(p>0.05)$. However, in VEGFB (rs12366035: C/T), VEFGC (rs7664413: C/T), and VEGFR1 (rs7993418: $\mathrm{A} / \mathrm{G}$ ) variants, genotype frequencies deviated from HWE. Since both groups that were under investigation in the present study were not selected from the general population but consisted of hospital-based selected patients with diabetes, the aforementioned variants were not excluded from analyses. Moreover, the high quality of assays "call rate $=100 \%$ with unambiguous allelic discrimination plots" suggested a violation of HWE assumptions in the study groups rather than technical genotyping errors [24].

On the comparison between all diabetic patients and controls, differential genotype and allele frequencies were observed in VEGFB (rs12366035: C/T), VEFGC (rs7664413: C/T), and VEGFR1 (rs7993418: A/G) vari- 
Fig. 2. Adjusted genetic association models for risk assessment to develop $\mathrm{DM}(\mathbf{a}-\mathbf{e})$ and DR $(\mathbf{f}-\mathbf{j})$. The two-sided $\chi^{2}$ test was used. Adjusted OR and $95 \%$ CI by sex and age are presented in the figure. Regression analysis revealed that the $\mathrm{rs} 12366035^{*} \mathrm{~T}$ variant of $V E G F B$ gene increased the susceptibility for developing DM (b) (allelic model [ $\mathrm{T}$ vs. $\mathrm{C}]: \mathrm{OR}=1.76$ and $95 \% \mathrm{CI}=1.15-2.70$; homozygote comparison [T/T vs. C/C]: $\mathrm{OR}=3.64$ and $95 \% \mathrm{CI}=1.42-9.34$; and recessive model $[\mathrm{T} / \mathrm{T}$ vs. $\mathrm{C} / \mathrm{C}+\mathrm{C} / \mathrm{T}]$ : $\mathrm{OR}=3.77$ and $95 \% \mathrm{CI}=1.50-9.50$ ). Likewise, subjects with the rs7664413*T variant of VEGFC gene were more likely to develop DM (c) (allelic model [T vs. $\mathrm{C}]: \mathrm{OR}=2.23$ and $95 \% \mathrm{CI}=1.40$ 3.59; homozygote comparison [T/T vs. $\mathrm{C} / \mathrm{C}]: \mathrm{OR}=2.59$ and $95 \% \mathrm{CI}=1.00$ 6.68 ; and dominant model [T/T vs. C/C $+\mathrm{C} / \mathrm{T}]: \mathrm{OR}=2.06$ and $95 \% \mathrm{CI}=1.14$ 3.75). The same 2 variants also conferred higher risk for DR progression under the allelic model (OR $=1.71,95 \%$ $\mathrm{CI}=1.07-2.74)$, homozygote comparison $(\mathrm{OR}=3.55,95 \% \mathrm{CI}=1.32-9.57)$, and recessive model $(\mathrm{OR}=3.77,95 \%$ $\mathrm{CI}=1.43-9.93$ ) for $V E G F B$ rs 12366035 polymorphism (g) and under the allelic model $(\mathrm{OR}=2.09,95 \% \mathrm{CI}=1.25-3.49)$, homozygote comparison $(\mathrm{OR}=2.76$, $95 \% \mathrm{CI}=1.02-7.45)$, and recessive model $(\mathrm{OR}=2.62,95 \% \mathrm{CI}=0.98-6.98)$ for VEGFC rs7664413 SNP (h). In contrast, VEGFR1 rs7993418 conferred protection against DR under heterozygote comparison (A/G vs. $A / A)$ and dominant model (G/G vs. A/A + A/G), as depicted in (i). DM, diabetes mellitus; DR, diabetic retinopathy; OR, odds ratio; 95\% CI, 95\% confidence interval. * Significant result.
Risk for developing DM rs833069 (T/C)

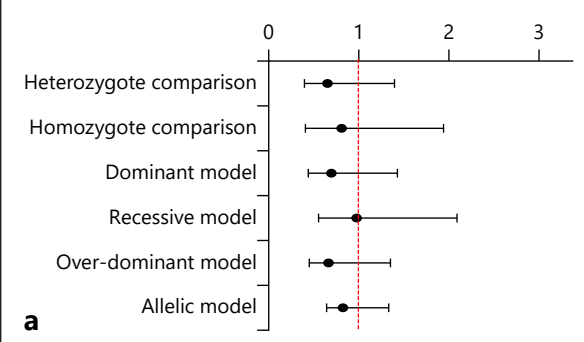

rs12366035 (C/T)

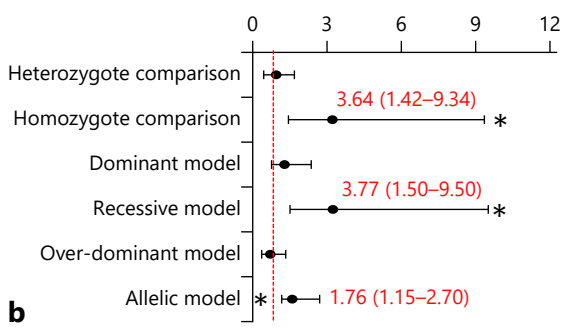

rs7664413 (C/T)

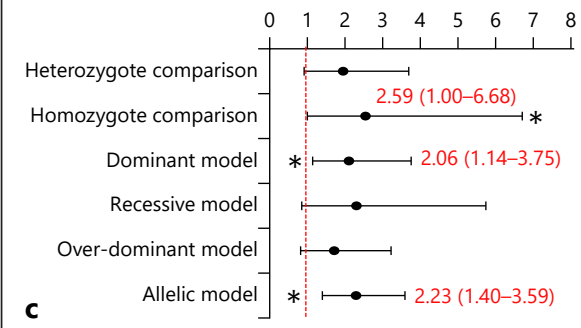

C

rs7993418 (A/G)

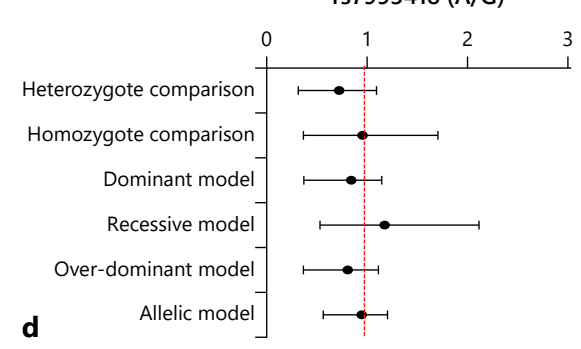

rs2305948 (C/T)

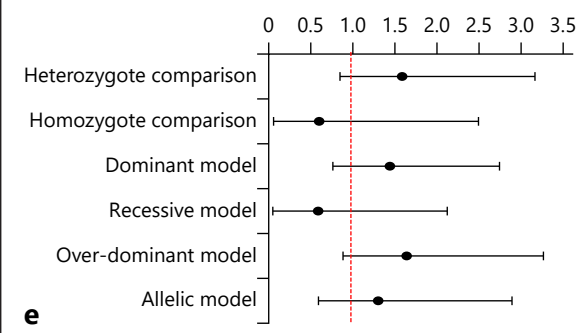

Risk for developing DR

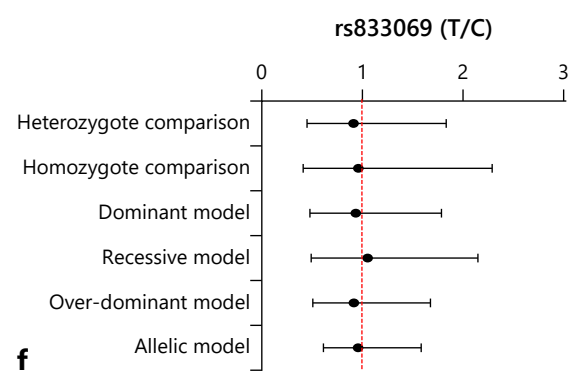

rs12366035 (C/T)

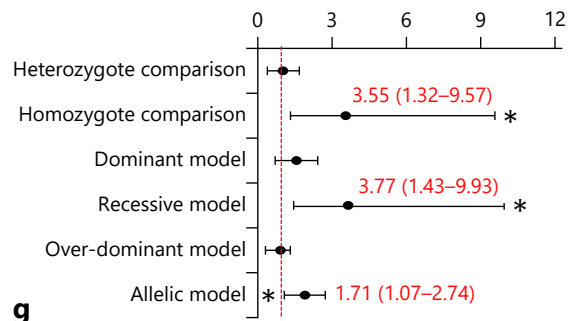

rs7664413 (C/T)

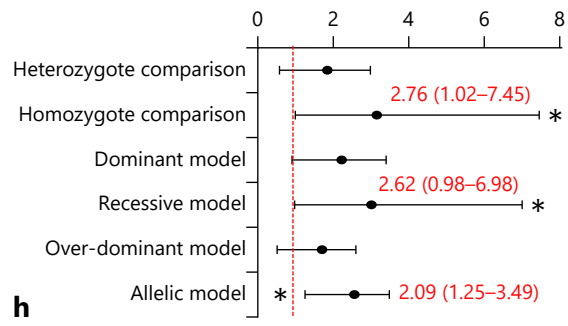

rs7993418 (A/G)

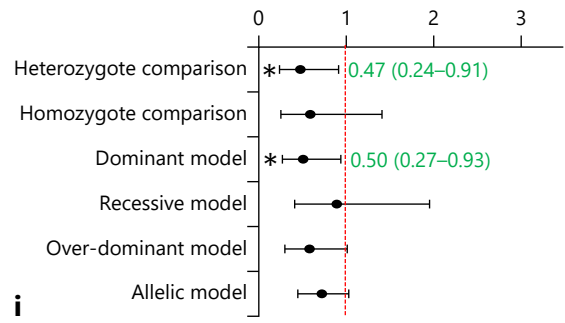

rs2305948 (C/T)

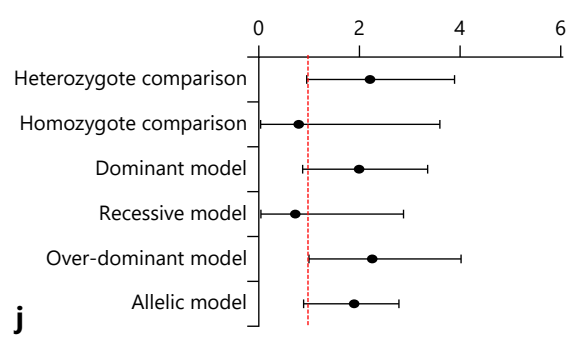




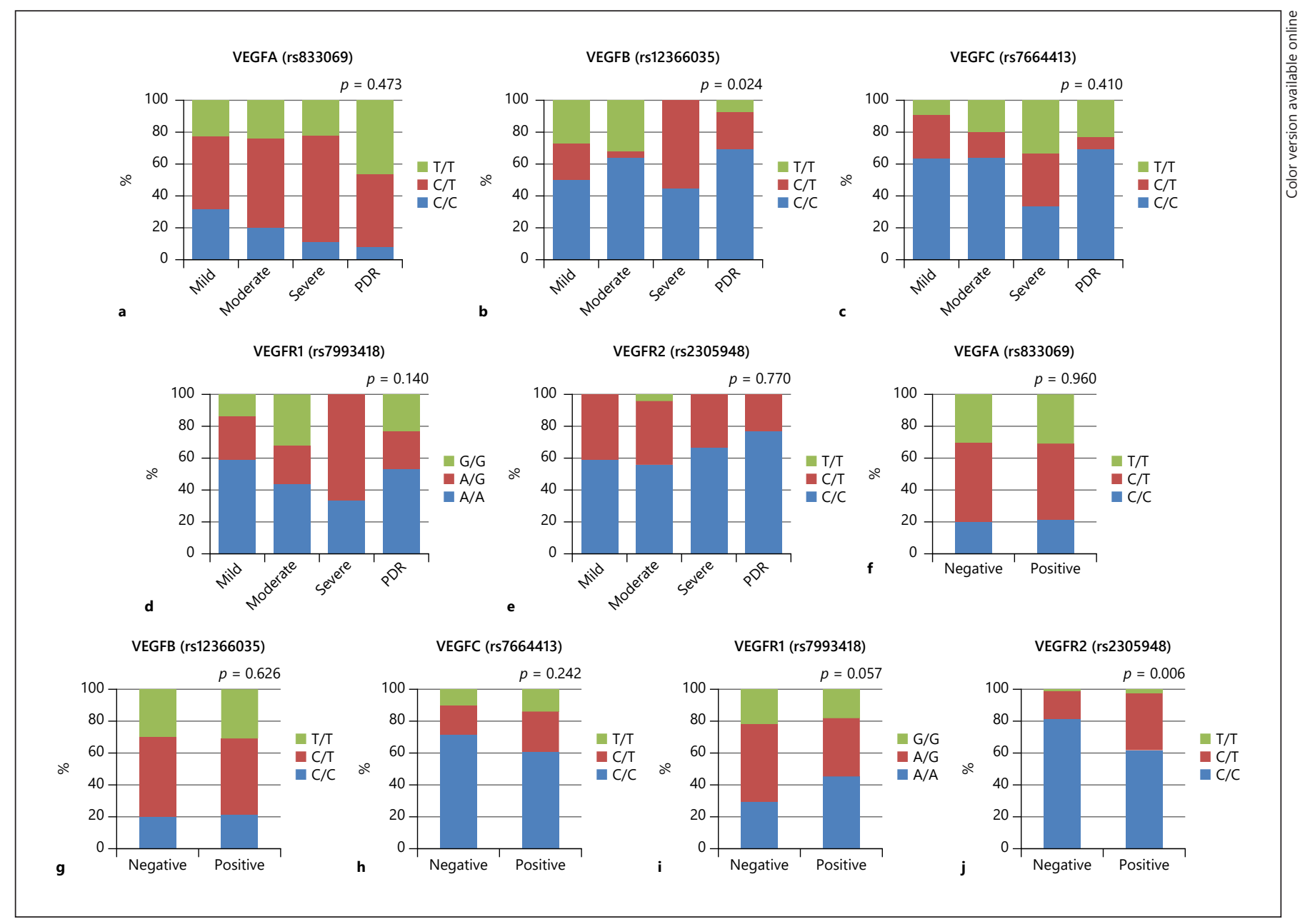

Fig. 3. Association of genotypes with clinical characteristics in DR patients: according to disease grade (a-e) and according to the presence of hypertension (nongenetic risk factor) $(\mathbf{f}-\mathbf{j})$. A $\chi^{2}$ test was used for the overall comparison. $p$ value $<0.05$ was considered as statistically significant. DR, diabetic retinopathy; PDR, proliferative diabetic retinopathy.

ants (Table 2). Higher frequencies of rs $12366035^{*} \mathrm{~T} / \mathrm{T}$ and rs7664413*T/T homozygosity were identified among patients compared to controls ( $p=0.014$ and 0.012 , respectively). Consistently, the minor allele frequencies ( $\mathrm{T}$ allele) were more prevalent among patient cohorts compared to the control group ( 31 vs. $20 \%$ in rs 12366035 $[p=0.008]$ and 28 vs. $15 \%[p<0.001])$. For VEGFR1 SNP (rs7993418: A/G), the A/G genotype was significantly lower in patients $(38 \%)$ versus controls $(54 \%)(p<0.001)$ (Table 2). In contrast, no association was found between genotype frequencies of both VEGFA (rs833069: T/C) and VEGFR2 (rs2305948: C/T) and diabetic disease. Comparison between the subset of diabetic patients who developed retinopathy and control subjects revealed similar findings; rs $12366035^{*} \mathrm{~T}$ and $\mathrm{rs} 7664413^{*} \mathrm{~T}$ variants were more prevalent among DR patients $(p=0.023$ and $0.004)$, while heterozygosity of rs7993418*A/G was less frequent among DR cohorts $(p=0.020)$ (Table 2).

After adjustment by sex and age, genetic association models (Fig. 2) revealed that the rs $12366035^{*} \mathrm{~T}$ variant of VEGFB gene and rs7664413*T variant of VEGFC gene increased the susceptibility for developing DM (Fig. 2b, c). The same 2 variants also conferred a higher risk for DR progression (Fig. 2g, h). In contrast, VEGFR1 rs7993418 conferred protection against DR, as depicted in Figure $2 \mathrm{i}$.

\section{Association of VEGF Gene Family Variants with Clinical Features}

Patients with PDR had a high frequency of $\mathrm{C} / \mathrm{C}$ genotype (69.2\%) of the VEGFB variant compared to T/T gen- 
Table 3. Association of VEGF gene family genotypes with ophthalmological assessments

\begin{tabular}{lllllll}
\hline SNP & Pre-CMT & Post-CMT & $\Delta$ CMT & Pre-BCVA & Post-BCVA & $\Delta$ BCVA \\
\hline rs833069 & 0.188 & 0.507 & 0.212 & 0.037 & 0.031 & 0.539 \\
rs12366035 & 0.333 & 0.585 & 0.694 & 0.008 & 0.086 & 0.085 \\
rs7664413 & 0.536 & 0.362 & 0.460 & 0.284 & 0.692 & 0.651 \\
rs7993418 & 0.054 & 0.821 & 0.037 & 0.668 & 0.486 & 0.204 \\
rs2305948 & 0.665 & 0.069 & 0.404 & 0.928 & 0.877 & 0.066 \\
\hline
\end{tabular}

Data are presented as $p$ values of association. The Kruskal-Wallis test was used. VEGF, vascular endothelial growth factor; CMT, central macular thickness; Pre, pretreatment with aflibercept; Post, posttreatment with aflibercept; $\triangle \mathrm{CMT}$, change in CMT; BCVA, best-corrected visual acuity assessed by the logarithm of the minimum angle of resolution. $p$ values $<0.05$ are considered significant.

otype (7.7\%) (Fig. 3a-e). Also, carriers of rs $2305948^{*} \mathrm{C} / \mathrm{T}$ of VEGFR2 had higher frequencies of hypertension disease (35.4\% in hypertensive patients vs. $17.9 \%$ in nonhypertensive diabetic cohorts, $p=0.006$ ) (Fig. $3 \mathrm{f}-\mathrm{j}$ ).

Patients with rs7993418*G/G of VEGFR1 gene exhibited a significant reduction in CMT change after aflibercept injection $(12.26 \pm 35.43 \mu \mathrm{m}$ in $\mathrm{G} / \mathrm{G}$ vs. $3.57 \pm 8.74 \mu \mathrm{m}$ in $\mathrm{A} / \mathrm{A})(p=0.037)$. VEGFA (rs833069) was associated with baseline BCVA level $(0.63 \pm 0.27$ in $\mathrm{T} / \mathrm{T}$ vs. $0.40 \pm$ 0.24 in $\mathrm{C} / \mathrm{C})(p=0.037)$ and posttreatment BCVA $(0.45$ \pm 0.27 for T/T vs. $0.23 \pm 0.16$ for $\mathrm{C} / \mathrm{C})(p=0.031)$. Nevertheless, homozygote patients with rs $12366035^{*} \mathrm{~T} / \mathrm{T}$ genotype (VEGFB) had the worst pretreatment BCVA score $(0.35 \pm 0.24)$ compared to other corresponding genotypes whose mean BCVA scores were $0.66 \pm 0.26$ in $\mathrm{C} / \mathrm{T}$ and $0.54 \pm 0.25$ in $\mathrm{C} / \mathrm{C}$ carriers $(p=0.008)$ (Table 3 ).

\section{Functional Enrichment Analysis}

The protein-protein interaction of VEGF protein family members is illustrated in online suppl. Figure 2. VEGF protein family members are enriched in several KEGG and Reactome pathways associated with DM and DR such as advanced glycation end-product-receptor of AGE (AGE-RAGE) signaling pathway in diabetic complications, phosphatidylinositol 3-kinase (PI3K) and Akt/protein kinase B signaling pathway, cellular responses to stress, and mitogen-activated protein kinase (MAPK) signaling pathway.

\section{Ligand-Receptor Interactions}

A summary of ligand-receptor interactions of their cocrystallized ligands is shown in online suppl. Figure 3, demonstrating the interacting key amino acid residues (as molecular targets) for maintaining their biological activities.

VEGF Gene Family Variants and Diabetic Retinopathy

\section{Discussion}

$\mathrm{DR}$ is one of the most prevalent microvascular complications of T2DM with variable frequencies in different ethnicities [15]. Although advancement in anti-VEGF therapy revealed significant clinical benefits in DR patients, the majority of patients failed to achieve clinically remarkable visual improvement. In this sense, further screening of the underlying molecular mechanisms may provide targets for early interventions and implementation of more extensive preventive strategies [25].

The current study indicates an association between $V E G F$ family members/receptor variants and DR susceptibility and/or aflibercept treatment response. More specifically, we found for the first time that carrying of VEGFR1 rs7993418 A/G genotype confers protection for the development of DR and associates with good aflibercept treatment outcomes in terms of anatomical improvement in CMT. Although, both rs12366035*T and rs7664413*T variants of $V E G F B$ and $V E G F C$ genes, respectively, increased the susceptibility for developing DM/DR, only homozygote patients for the minor allele of the former gene demonstrated the worst pre-treatment BCVA score compared to other corresponding genotypes. These findings, consistent with the recent speculation of Cobos et al. [26], that "the variable response to anti-VEGF treatment observed in clinical practice," could be explained by genotype.

It is well known that VEGF plays an important role in $\mathrm{DM} / \mathrm{DR}$ pathogenesis [27]. The degree of DME is directly related to VEGF levels, which increase the abnormal angiogenesis and the permeability of the blood-retinal barrier, leading to macular edema [2]. Hence, it is not surprising that the VEGF family and its receptors could be putative biomarkers in DR pharmacogenetics. Several studies 
have investigated the impact of multiple genetic variants of the VEGF family on either DR susceptibility $[15,17$, 27-31] or the response to treatment for anti-VEGF therapy $[14,32]$, though the results were inconclusive yet.

The first VEGF gene variant that we studied is the intronic VEGFA rs833069 that is located on chromosome $6 \mathrm{p} 21.1$. The product of this gene was the first described member of the VEGF family, which induces angiogenesis by activating the related tyrosine kinase receptors (TKR), "VEGFR1 and VEGFR2," on endothelial cells [33]. Although we did not find an association of this variant with $\mathrm{DM} / \mathrm{DR}$ susceptibility, it was significantly associated with baseline and posttreatment BCVA levels. This polymorphism has been associated previously with AMD development [34], as well as with the anatomic response to IV anti-VEGF therapy in the same type of patients [35]. Also, this variant was associated with increased risk for DR in type $1 \mathrm{DM}$ [36] and the severity of T2DM-related DR [32]. Meanwhile, it did not show a significant association with the patients' responses to anti-VEGF therapy [32]. The differences in study design, sample size, collection and type of samples, participant ethnicities, and detection methods could participate in the observed contradictory results.

The second VEGFB variant (rs12366035) is located on chromosome 11q13.1. It has been found that circulating VEGFB levels associated with DR [37], and targeting VEGFB signaling in rodent models of T2DM, showed improvement in insulin resistance [38]. Several polymorphisms in this gene have been analyzed with DR or severe DR [28,39-41]. In addition, recent work by Ung et al. [42] had identified 3 nonsynonymous variants (rs138325963, rs61384522, and 1 novel variant) in this gene, using whole exome sequencing, which could be related to PDR [43].

The third current variant is the intronic rs7664413 VEGFC polymorphism on chromosome 4q34.3. VEGFC has displayed a "high degree of homology" with VEGFA among other VEGF family members [43]. Zhao et al. [43] have revealed that tumor necrosis factor-alpha and prolonged hyperglycemia could stimulate VEGFC expression in retinal endothelial cells, which in turn interact with VEGFR-2 to sustain "retinal neovascularization." To our knowledge, there were no reports concerning the role of the VEGFC rs7664413 variant in DR or response to anti-VEGF therapy.

Although the functional importance of this intronic variant on gene expression has not been tested experimentally, previous literature indicated that "if the intronic SNP of a gene was detected in the RNA extract, the allelic gene expression could yield very similar estimates to those obtained with exonic SNPs" [44]. Several studies also reported that "an intronic SNP can affect gene expressions" $[45,46]$. The authors speculated that this intronic variant might affect VEGFC mRNA expression level that leads to high protein levels supporting the observed association of the rs7664413*T/T homozygosity with DM and DR in the current study. Hsieh et al. [47] also suggested this variant might also affect the premessenger RNA splicing. Notably, the aforementioned assumptions will need to be tested experimentally in future studies to be validated.

Another essential anti-VEGF-related gene is VEGFR1. This gene is located in chromosome 13q12.3 and encodes for a TKR that mediates signal transduction cascades from several members of the VEGF family (Fig. 1b) and plays an essential role in angiogenesis, especially in pathological conditions [48]. Hence, it is plausible that variants within VEGFR-related genes could predict response to antiVEGF therapy. Although no previous reports were correlating this variant VEGFR1 rs7993418 directly to DR, consistent with our findings, Cobos et al. [26] reported an association of this SNP with good anatomical outcome after IV ranibizumab treatment in neovascular AMD patients. Replication studies on DR cohorts with different ethnicities are necessary to confirm this association.

The last variant we studied is the missense VEGFR2 rs2305948, which is located on chromosome 4q12. By screening of previously published literature (https:// www.ncbi.nlm.nih.gov/snp/rs2305948\#publications), this SNP has not been associated in prior genetic studies with DR and/or anti-VEGF therapy.

Although, to our knowledge, we paid attention for the first time to the impact of some VEGF-related variants that have not been previously identified in other "candidate gene studies" in DM/DR population cohorts, the relatively small sample size and the study design that is confined to a single ethnic group (Middle East), as well as the lack of in vitro functional validation studies, could limit this analysis. Hence, further larger sample size and longterm follow-up replication studies with confirmatory laboratory quantitative analyses on diabetic animal models would be necessary to confirm the association between the study variants and DR/response to anti-VEGF therapy.

\section{Conclusions}

This study revealed significant associations of some VEGF-related polymorphisms: VEGFB rs12366035*T, VEGFC rs7664413*T, and VEGFR1 rs7993418 A/G with $\mathrm{DM} / \mathrm{DR}$ susceptibility. Also, some variants showed an as- 
sociation with good aflibercept treatment early outcome. These variants could be good candidates for future pharmacogenetic studies after their functional validation.

\section{Acknowledgements}

The authors thank the Center of Excellence in Molecular and Cellular Medicine and Oncology Diagnostic Unit, Suez Canal University, Ismailia, Egypt, for providing the facilities for performing the research work, and they also thank all participants who agree to participate in the current study.

\section{Statement of Ethics}

All procedures performed in studies involving human participants were in accordance with the ethical standards of the institutional and/or national research committee and with the $1964 \mathrm{Hel}-$ sinki Declaration and its later amendments or comparable ethical standards. The institutional research ethics committee of the Faculty of Medicine, Suez Canal University, (Approval No. 3648) approved the study. Written informed consent was obtained from all the study participants before taking part.

\section{Conflict of Interest Statement}

The authors have no conflicts of interest to declare.

\section{Funding Sources}

This research did not receive any specific grant from funding agencies in the public, commercial, or not-for-profit sectors.

\section{Author Contributions}

A.A., E.A.T., A.A.M., R.M.L., M.H.S., and M.S.F. conceived and designed the experiments. A.A. and A.A.M. recruited the study samples and clinical patient data with the ethical application. E.A.T. and M.S.F. contributed to parts of the molecular experiments. E.A.T. contributed to the statistical analysis. M.S.N. contributed to the evaluation of the ligand-receptor interaction. All authors contributed the reagents and materials needed for the current work and contributed to writing, reading, and approval of the final manuscript.

\section{References}

1 Lee R, Wong TY, Sabanayagam C. Epidemiology of diabetic retinopathy, diabetic macular edema and related vision loss. Eye Vis. 2015; $2: 17$.

2 Duh EJ, Sun JK, Stitt AW. Diabetic retinopathy: current understanding, mechanisms, and treatment strategies. JCI Insight. 2017 Jul; 2(14): e93751.

3 Boyer DS, Hopkins JJ, Sorof J, Ehrlich JS. Anti-vascular endothelial growth factor therapy for diabetic macular edema. Ther Adv Endocrinol Metab. 2013 Dec;4(6):151-69.

4 Colucciello M. Current intravitreal pharmacologic therapies for diabetic macular edema. Postgrad Med. 2015 Aug;127(6):640-53.

5 Fogli S, Del Re M, Rofi E, Posarelli C, Figus M, Danesi R. Clinical pharmacology of intravitreal anti-VEGF drugs. Eye. 2018 Jun;32(6): 1010-20.

6 Elman MJ, Elman MJ, Aiello LP, Beck RW, Bressler NM, Bressler SB, et al. Randomized trial evaluating ranibizumab plus prompt or deferred laser or triamcinolone plus prompt laser for diabetic macular edema. Ophthalmology. 2010 Jun;117(6):1064-77.e35.

7 Heier JS, Korobelnik JF, Brown DM, SchmidtErfurth U, Do DV, Midena E, et al. Intravitreal aflibercept for diabetic macular edema: 148-week results from the VISTA and VIVID studies. Ophthalmology. 2016 Nov;123(11): 2376-85.

8 Wells JA, Glassman AR, Ayala AR, Jampol LM, Bressler NM, Bressler SB, et al. Aflibercept, bev- acizumab, or ranibizumab for diabetic macular edema: two-year results from a comparative effectiveness randomized clinical trial. Ophthalmology. 2016 Jun;123(6):1351-9.

9 Stewart MW, Rosenfeld PJ. Predicted biological activity of intravitreal VEGF trap. Br J Ophthalmol. 2008 May;92(5):667-8.

10 Korobelnik JF, Do DV, Schmidt-Erfurth U, Boyer DS, Holz FG, Heier JS, et al. Intravitreal aflibercept for diabetic macular edema. Ophthalmology. 2014 Nov; 121(11):2247-54.

11 Cornel S, Adriana ID, Mihaela TC, Speranta S, Algerino S, Mehdi B, et al. Anti-vascular endothelial growth factor indications in ocular disease. Rom J Ophthalmol. 2015 OctDec;59(4):235-42.

12 Deák GG, Bolz M, Ritter M, Prager S, Benesch T, Schmidt-Erfurth U, et al. A systematic correlation between morphology and functional alterations in diabetic macular edema. Invest Ophthalmol Vis Sci. 2010 Dec;51(12):6710-4.

13 Yang X, Deng Y, Gu H, Lim A, Altankhuyag A, Jia W, et al. Polymorphisms in the vascular endothelial growth factor gene and the risk of diabetic retinopathy in Chinese patients with type 2 diabetes. Mol Vis. 2011;17:3088-96.

14 El-Shazly SF, El-Bradey MH, Tameesh MK. Vascular endothelial growth factor gene polymorphism prevalence in patients with diabetic macular oedema and its correlation with anti-vascular endothelial growth factor treatment outcomes. Clin Exp Ophthalmol. 2014 May-Jun;42(4):369-78.
15 Han L, Zhang L, Xing W, Zhuo R, Lin X, Hao $Y$, et al. The associations between VEGF gene polymorphisms and diabetic retinopathy susceptibility: a meta-analysis of 11 case-control studies. J Diabetes Res. 2014;2014:805801.

16 Wang H, Cheng JW, Zhu LS, Wei RL, Cai JP, $\mathrm{Li} \mathrm{Y}$, et al. Meta-analysis of association between the $-2578 \mathrm{C} / \mathrm{A}$ polymorphism of the vascular endothelial growth factor and retinopathy in type 2 diabetes in Asians and Caucasians. Ophthalmic Res. 2014;52(1):1-8.

17 Kaidonis G, Burdon KP, Gillies MC, Abhary S, Essex RW, Chang JH, et al. Common sequence variation in the VEGFC gene is associated with diabetic retinopathy and diabetic macular edema. Ophthalmology. 2015 Sep; 122(9):1828-36.

18 Early treatment diabetic retinopathy study design and baseline patient characteristics. ETDRS report number 7. Ophthalmology. 1991 May;98(5 Suppl):741-56.

19 Toraih EA, Abdelghany AA, Abd El Fadeal NM, Al Ageeli E, Fawzy MS. Deciphering the role of circulating lncRNAs: RNCR2, NEAT2, CDKN2B-AS1, and PVT1 and the possible prediction of anti-VEGF treatment outcomes in diabetic retinopathy patients. Graefes Arch Clin Exp Ophthalmol. 2019 Sep;257(9):1897-913.

20 Hussein MH, Toraih EA, Aly NM, Riad E Fawzy MS. A passenger strand variant in miR-196a2 contributes to asthma severity in children and adolescents: a preliminary study. Biochem Cell Biol. 2016 Aug;94(4):347-57. 
21 Pettersen EF, Goddard TD, Huang CC, Couch GS, Greenblatt DM, Meng EC, et al. UCSF Chimera--a visualization system for exploratory research and analysis. J Comput Chem. 2004 Oct;25(13):1605-12.

22 Bissantz C, Kuhn B, Stahl M. A medicinal chemist's guide to molecular interactions. J Med Chem. 2010 Jul;53(14):5061-84.

23 Toraih EA, Ismail NM, Toraih AA, Hussein MH, Fawzy MS. Precursor miR-499a variant but not miR-196a2 is associated with rheumatoid arthritis susceptibility in an Egyptian population. Mol Diagn Ther. 2016 Jun;20(3): 279-95.

24 Esser C, Tomluk J. Reporting Hardy-Weinberg tests in case-control studies: reasons for caution but not for panic reactions. J Invest Dermatol. 2005 May;124(5):1082-3.

25 Wang W, Lo ACY. Diabetic retinopathy: pathophysiology and treatments. Int J Mol Sci. 2018 Jun;19(6):1816.

26 Cobos E, Recalde S, Anter J, Hernandez-Sanchez M, Barreales C, Olavarrieta L, et al. Association between CFH, CFB, ARMS2, SERPINF1, VEGFR1 and VEGF polymorphisms and anatomical and functional response to ranibizumab treatment in neovascular agerelated macular degeneration. Acta Ophthalmol. 2018 Mar;96(2):e201-12.

27 Paine SK, Basu A, Mondal LK, Sen A, Choudhuri S, Chowdhury IH, et al. Association of vascular endothelial growth factor, transforming growth factor beta, and interferon gamma gene polymorphisms with proliferative diabetic retinopathy in patients with type 2 diabetes. Mol Vis. 2012;18:2749-57.

28 Abhary S, Burdon KP, Gupta A, Lake S, Selva $\mathrm{D}$, Petrovsky N, et al. Common sequence variation in the VEGFA gene predicts risk of diabetic retinopathy. Invest Ophthalmol Vis Sci. 2009 Dec;50(12):5552-8.

29 Lu Y, Ge Y, Shi Y, Yin J, Huang Z. Two polymorphisms (rs699947, rs2010963) in the VEGFA gene and diabetic retinopathy: an updated meta-analysis. BMC Ophthalmol. 2013 Oct; 13:56.

30 Fan X, Wu Q, Li Y, Hao Y, Ning N, Kang Z, et al. Association of polymorphisms in the vascular endothelial growth factor gene and its serum levels with diabetic retinopathy in
Chinese patients with type 2 diabetes: a crosssectional study. Chin Med J. 2014;127(4): 651-7.

31 Xie XJ, Yang YM, Jiang JK, Lu YQ. Association between the vascular endothelial growth factor single nucleotide polymorphisms and diabetic retinopathy risk: a meta-analysis. J Diabetes. 2017 Aug;9(8):738-53.

32 Tetikoğlu M, Yüksel Z, Aktas S, Sağdik HM, Özcura F. VEGF-A gene polymorphisms and responses to intravitreal ranibizumab treatment in patients with diabetic macular edema. Int Ophthalmol. 2018 Dec;38(6): 2381-8.

33 Rahimi N. Vascular endothelial growth factor receptors: molecular mechanisms of activation and therapeutic potentials. Exp Eye Res. 2006 Nov;83(5):1005-16.

34 Galan A, Ferlin A, Caretti L, Buson G, Sato G, Frigo AC, et al. Association of age-related macular degeneration with polymorphisms in vascular endothelial growth factor and its receptor. Ophthalmology. 2010 Sep;117(9): 1769-74.

35 Chang W, Noh DH, Sagong M, Kim IT. Pharmacogenetic association with early response to intravitreal ranibizumab for age-related macular degeneration in a Korean population. Mol Vis. 2013;19:702-9.

36 Al-Kateb H, Mirea L, Xie X, Sun L, Liu M, Chen $\mathrm{H}$, et al. Multiple variants in vascular endothelial growth factor (VEGFA) are risk factors for time to severe retinopathy in type 1 diabetes: the DCCT/EDIC genetics study. Diabetes. 2007 Aug;56(8):2161-8.

37 Sun CY, Lee CC, Hsieh MF, Chen CH, Chou KM. Clinical association of circulating VEGF$\mathrm{B}$ levels with hyperlipidemia and target organ damage in type 2 diabetic patients. J Biol Regul Homeost Agents. 2014 Apr-Jun;28(2): 225-36.

38 Hagberg CE, Mehlem A, Falkevall A, Muhl L, Fam BC, Ortsäter $\mathrm{H}$, et al. Targeting VEGF-B as a novel treatment for insulin resistance and type 2 diabetes. Nature. 2012 Oct;490(7420): 426-30.

39 Awata $\mathrm{T}$, Inoue $\mathrm{K}$, Kurihara $\mathrm{S}$, Ohkubo $\mathrm{T}$, Watanabe $\mathrm{M}$, Inukai K, et al. A common polymorphism in the $5^{\prime}$-untranslated region of the VEGF gene is associated with diabetic reti- nopathy in type 2 diabetes. Diabetes. 2002 May;51(5):1635-9.

40 Abhary S, Hewitt AW, Burdon KP, Craig JE. A systematic meta-analysis of genetic association studies for diabetic retinopathy. Diabetes. 2009 Sep;58(9):2137-47.

41 Zhao T, Zhao J. Association between the $-634 \mathrm{C} / \mathrm{G}$ polymorphisms of the vascular endothelial growth factor and retinopathy in type 2 diabetes: a meta-analysis. Diabetes Res Clin Pract. 2010 Oct;90(1):45-53.

42 Ung C, Sanchez AV, Shen L, Davoudi S, Ahmadi T, Navarro-Gomez D, et al. Whole exome sequencing identification of novel candidate genes in patients with proliferative diabetic retinopathy. Vision Res. 2017 Oct; 139:168-76.

43 Zhao B, Smith G, Cai J, Ma A, Boulton M. Vascular endothelial growth factor C promotes survival of retinal vascular endothelial cells via vascular endothelial growth factor receptor-2. Br J Ophthalmol. 2007 Apr;91(4): $538-45$.

44 Serre D, Gurd S, Ge B, Sladek R, Sinnett D, Harmsen E, et al. Differential allelic expression in the human genome: a robust approach to identify genetic and epigenetic cis-acting mechanisms regulating gene expression. PLoS Genet. 2008 Feb;4(2):e1000006.

45 Tsukada S, Tanaka Y, Maegawa H, Kashiwagi A, Kawamori R, Maeda S. Intronic polymorphisms within TFAP2B regulate transcriptional activity and affect adipocytokine gene expression in differentiated adipocytes. Mol Endocrinol. 2006 May;20(5):1104-11.

46 Wang D, Guo Y, Wrighton SA, Cooke GE Sadee W. Intronic polymorphism in CYP3A4 affects hepatic expression and response to statin drugs. Pharmacogenomics J. 2011 Aug; 11(4):274-86.

47 Hsieh MC, Hsu HT, Hsiao PC, Yang SF, Yeh $\mathrm{CB}$, Bien MY, et al. Role of VEGF-C gene polymorphisms in susceptibility to hepatocellular carcinoma and its pathological development. J Clin Lab Anal. 2014 May;28(3):237-44.

48 Cross MJ, Claesson-Welsh L. FGF and VEGF function in angiogenesis: signalling pathways, biological responses and therapeutic inhibition. Trends Pharmacol Sci. 2001 Apr;22(4): 201-7. 